\title{
Coronavirus Disease 19 and Severe Acute Respiratory Syndrome Coronavirus 2: To More Efficient Control
}

\author{
Otmane El Brini
}

\section{ABSTRACT}

The coronavirus disease 19 (COVID-19) is a viral infection caused by a new coronavirus named severe acute respiratory syndrome coronavirus 2 (SARS$\mathrm{CoV}-2$ ). It is considered as a public health emergency of international concern. The only available way, apparently effective, to control this pandemic is the social distancing. However, other possible aspects of infection control may be mentioned. In this review, we highlight certain peculiarities linked to the nature of the virus and its angiotensin converting enzyme 2 receptor, the host defense and the final clinical manifestation of the infection that could be essential elements in strengthening the effectiveness of COVID-19 pandemic control efforts.

Keywords: Severe acute respiratory syndrome coronavirus 2, Coronavirus disease 19, Angiotensin converting enzyme 2, Pandemic.

Published Online: May 30, 2020
ISSN: 2684-5199
DOI : 10.24018/ejbio.2020.1.3.29
Otmane EL BRINI *
National Laboratory of Genetic,
Neuroendocrinology and
Biotechnology. Ibn Tofail University.
Faculty of Science. Kenitra. Morocco.
(e-mail: otmanee @hotmail.com )
Laboratory of Medical Analysis.
Regional Hospital Center. Ministry of
Health. Morocco.
(e-mail: otmaner @hotmail.com)
*Corresponding Author

\section{INTRODUCTION}

Since December, a mass pneumonia of unknown origin has occurred in Wuhan, the capital city of Hubei province, China [1]. The pathogen involved, was then isolated and identified as a new coronavirus first named 2019 novel coronavirus (2019-nCov) [2]. It was later named severe acute respiratory syndrome coronavirus 2 (SARS-CoV-2) [3] and the corresponding pneumonia corona virus disease 19 (COVID-19). Because of the great spread of the disease in China and all over the world and the deaths caused aroused the world health Organization (WHO) to consider the COVID-19 as a public health emergency of international concern [1]. In infectious diseases, whether epidemic or not, to control the infection, must to control its origine, to interrupt the transmission chain and to defend people at risk. In COVID-19, it is not yet possible to protect the susceptible population because of the difficulty, until now, to develop a safe and effective vaccine against the disease. It is thus believed that the only means that remains within reach is the social distancing established by most of the countries affected by this new pandemic. However, other possible aspects of infection control may be mentioned.

The objective of this review is to emphasize the particularities associated with COVID-19 and its pathogenic agent which must be taken into consideration to consolidate the control approaches already taken.

\section{THE SOURCE OF COVID-19 INFECTION}

The pathogen responsible for the COVID-19 pandemic is an enveloped, single strand, positive sense RNA $\beta$ coronavirus [4]. It belongs to the Coronaviridae family whose genome lenght varies from 26 to 32 kilobases $(\mathrm{kb})$ [4]. The genomic sequences of SARS-CoV-2 isolates in people with COVID-19 were $29.9 \mathrm{~kb}$ in length [5].

All coronaviruses show similarities in their genomic expression and organization. Nonstructural proteins are encoded by the Open reading Frame (ORF) $1 \mathrm{a} / \mathrm{b}$ at the 5, end, while the other ORFs encode for structural proteins, spike (S), envelope (E), membrane (M) and nucleocapside (N) at the 3' end [4], [6]. Remarkably, this organization is not fundamental for these viruses to form typically functional virions. Indeed, some coronaviruses do not necessarily require this range of structural proteins to become complete infectious virions [6]. This observation, presents the first peculiarity of these viruses, manifested in the mechanism of adaptation making what is structural as superfluous or by a mechanism coding other additional proteins with overlapping compenatory functions.

Pathogenically, all coronaviruses share the same organization at the ectodomain of their S proteins. It is made of two domains:

- $\quad$ The $\mathrm{S} 1$ domain, $\mathrm{N}$ terminal, diverges in sequences even among species of a single coroanvirus, functioning as a Receptor Binding Domain (RBD) and it is responsible for binding to a variety of proteins and sugars.

- $\quad$ The $\mathrm{S} 2$ domain, $\mathrm{C}$ terminal, most conserved region of the protein, and it is responsible for the fusion of the membranes of the viral particle and the host cell. 
In the case of SARS-CoV-2, the S protein (1273 amino acids) has a RBD mainly involved in the binding at its specific receptor, the Angiotensin Converting Enzyme 2 (ACE2) [7]. R. Lu et al showed that the RBD external subdomain of SARS-COV-2 is more similar to that of SARS-CoV [8]. Genomically, the RBD S prein of SARSCoV-2 was $73 \%$ - 76\% similar to that of the SARS-CoV from humain, civet or bat viruses [9]. Recently several binding structures SARS-CoV-2 S protein - ACE2 have been resolved, showing the main residues involved in this molecular interaction [7].

At the whole genomic level, SARS-CoV-2 was closer to SARS like coronaviruses, bat-SLCOVZXC21 and batSL-COVZC45 [8], [10]. Despite this genomic similarity, several arguments testify the presence of another animal functioning as an intermediate host between bats and humains [8]. The presence of this intermediate host emphasizes the power of 'jump species' - pathogenically significant - characterizing the coronaviruses. The latter, as typical RNA viruses, are capable of great genomic evolution because of their evolutionary rate of $10^{-4}$ nucleotide substitions per site per year [4]. In other words, they can accumulate 1 to 3 changes in their genome per month (rates of $3.10^{-4}$ to $1.10^{-3}$ par site per year) [11].

Interestingly, studies have shown that the genome of SARS-CoV-2 is not similar to that of severe acute respiratory syndrome coronavirus (SARS-CoV) or Middle East Respiratory Syndrome coronavirus (MERS-CoV) [8]. At the genomic level, six regions of difference exist between the SARS-CoV-2 and the SARS-CoV at genes ORF $1 \mathrm{a} / \mathrm{b}(3$ regions), $\mathrm{S}$ (2 regions) and ORF7b and ORF8 (1 region) [12]. These differences are reflected in a variation affecting 380 amino acid substitutions, including 5 of the 6 vital amino acids in the viral RBD S protein [5]. Surprisingly, among the 14 ACE2 contacting residues in the RBD, only 1 that has been substituted while 9 are totally preserved and 4 partially preserved between SARS-CoV-2 and SARS-CoV from human, civet and bat [9]. As result, the binding affinity of SARS-CoV-2 with its receptor ACE2 is less efficient than the 2002-2003 SARS-CoV but more efficient than the 20032004 SARS-CoV [13]. All of the alterations in the amino acid residues induces the formation of salt bridge and the improvement of hydrophobic virus-receptor interactions allowing the great spread of SARS-COV-2 and the resulting COVID-19 disease that the SARS epidemic [7]. Here again, another characteristic of the coronaviruses residing in the modification of their S proteins to adapt to their new host by mutations or recombinations with the coronaviruses already infecting this new host. In return, the circulation of coronaviruses in several susceptible hosts is capable of increasing the rate of recombination events of these viruses which creates a series of genetic diversity with unpredictable virulence, tropism and propagation power. In the W. Ren et al study, they showed that some SARS-CoV like viruses isolated from bats were not able to use the ACE2 receptor for entry into the host cell [14]. The replacement of the amino acid sequence between the residues 323 and 505 by a sequence from SARS-CoV RBD was sufficient to allow the use of this receptor by bat like coronaviruses [15]. Similarly, the adaptation of SARS-CoV to humans was due to two point mutations K79N and S487T in its binding domain [15]. In addition, Y. Wan et al were able to characterize specific amino acids in positions 442 , 472, 479, 480 and 487 capable of improving the binding of the virus to human ACE2 [9]. In an identical way, it has been predicted that a single mutation N501T (S487T mutation in SARS-CoV) may improve the binding affinity of SARS-CoV-2 RBD to its human ACE2 receptor [9]. The great power of coronaviruses to undergo genetic modifications is mainly due to the extraplasticity of its large RNA genome. This process allows the exchange of genetic material during co-infection as well as the accumulation of adaptive mutations. This dynamic and continuous evolution between the coronaviruses species and between coronaviruses and hosts allowed the realization of jump from bats to humans. This phenomenon is very powerful at the SARS-CoV-2, because it has been shown that to have most likely occurred in late November or early December 2019 [16]. The short time between the first cases of COVID19 infections and the isolation of the virus was sufficient to reveal several SARS-CoV-2 strains. In this context, it has been reported that 6 different genotypes have been observed in some COVID-19 patients [10]. In the phylogenetic analysis of isolated SARS-CoV-2 strains performed by $\mathrm{M}$. Hussain et al, showed the presence of two major types $\mathrm{S}$ and L (defined by just two SNPs in positions 8782 and 28144) [17]. They showed that $S$ type (the less frequent $~ 30 \%$ ) represents the oldest version of the virus and the less aggressive [17]. This result highlights the great adaptation of SARS-CoV-2 to the human host by promoting propagation rather than aggressiveness (by adapting its RBD). Another argument for this adaptation is the result of R. Lu et al study [8]. They showed that two environmental samples -isolated from the Huanan Market- belong to a single genomic type (type II) without any direct link to the other type (type I) with the Huanan Market [8]. This suggests that the virus has mutated in second level COVID-19 patients (COVID-19 patients infected by those with a direct link with Huanan Market and carrying the type II genotype of SARS-COV-2).

Faced with this permanent evolution of the SARS-CoV2 virus and its rapid transmission to a larger number of people, constant monitoring of mutations is mandatory and a comparison of symptoms with isolated strains is desirable.

\section{THE RECEPTOR OF THE SOURCE OF COVID-19 INFECTION}

The ACE2 is a key protease of the Renin Angiotensin System (RAS). It acts as a simple carboxypeptidase linked to the membrane capable of hydrolyzing the AngI to form the Ang (1-9) and the AngII to Ang(1-7) [18]. In vitro studies have shown that the catalytic efficiency of the ACE2 for the AngII is greater (400 fold) than for the AngI [19] which indicates that the major role of the ACE2 is the conversion of AngII into Ang(1-7). The latter represents an antagonist for the AT1R having antiproliferative and vasoprotective actions by a competitive counter action of the AngII [20]. The Ang(1-7) also bind to the Mas receptor. The ACE2-Ang(1-7)-Mas receptor axis exerts opposite actions to the activation of the AngII AT1R and can also promotes vasodilation [21]. The ACE2 gene, $39.98 \mathrm{~kb}$ in size, is located on chromosome Xp22 and contains 18 exons and 20 introns [18]. In reference to its viral receiving function, the 
lungs show a high ACE2 expression, identified in type II alveolar cells [22], [23]. In addition to the lungs, the respiratory tract (respiratory epithelial cells), oral tissue cells (tongue epithelial cells) have shown high expression of ACE2 [24]. These results demonstrate the increased sensitivity of these pathways and organs to COVID-19 and thus provide evidence on the possible routes of SARS-CoV2 entry into the human body. Moreover, several other organs express ACE2 including: esophagus [23], ileum [23], colon [23], myocardial cells [22], kidney [22], bladder [22], and intestines [24]. This observation may explain the nonrespiratory symptoms observed in COVID-19 patients. This is more evident in COVID-19 severe cases since the virus was able to damage even vital tissues expressing little ACE2. In this type of patient, generally with comorbidities, the molecular mechanism responsible for the severity of the disease is not fully understood but ACE2 has been suggested as a triggering factor [25]. Indeed, the expression of ACE2 in lung tissue is upregulated by comorbidities during COVID-19. It has been shown that the expression of ACE2 can increase in myocardial infarction [26], diabetes [27], heart failure [28], and complications of diabetes (such as cardiovascular and kidney diseases) [29], [30]. This finding suggests that the high incidence of COVID-19 in patients with comorbidities is mainly due to the increased expression of ACE2, SARS-CoV-2 receptor. Another argument in favor of this hypothesis comes from the fact that the treatment by RAS inhibitors as angiotensin-converting enzyme inhibitors (ACEI) or angiotensin-receptor blockers (ARBs) leads to the upregulation of ACE2 [9], [27]. In this context, several studies have shown that patients under these treatments manifest more severe COVID-19 symptoms with high mortality when compared with those without treatments [27]. From all of these results and since this treatment is frequently used in the case of diabetes and hypertension (HTA), special attention should be paid to people with these abnormalities in terms of prevention and education around the COVID-19 pandemic.

\section{DEFENSE AGAINST THE COVID-19 INFECTION}

Pathogens and hosts are in constant interaction to overpower each other. The viral pathogens cause a state of immunosuppression able of weakening and overwhelming the defense of the host according to the fitness of its immune system. Epidemiological studies have shown that male eldery individuals with chronic conditions are most affected by COVID-19 [31]. This pathological affinity has been attributed to the immune capital of the person concerned. Indeed, it has been shown that the immune response is subject to gender difference. Females develop a higher innate humoral and cellular immune response than males against viral infections [32]. On the one side, it was observed that the ACE2 pulmonary expression may be sex biased [33]. Both experimental and clinical evidences highlight an important influence of sex on the regulation of RAS and the subsequent cardiovascular diseases develpment. K. Chen et al have shown that ACE2 activity is increased in men than in women [34]. It thus appears that the male sex is a factor of susceptibility to COVID-19 following the reduction in the immune response coupled with the increase in ACE2 as receptor of SARS-CoV-2. On another side, aging as a complex biological process appears to be a predisposing factor for COVID-19. Indeed, immunosenescence associated with aging is characterized by an alteration and a decrease in the capacity of the immune system. Immune changes related to aging can be alterations in the number of monocytic and dendritic cells, limited diversity in the cell repertoire $\mathrm{B} / \mathrm{T}$, reduction in the phagocytic activity of neutrophils, epigenetic alterations, change in intracellular communication, genomic instability, cellular senescence and chronic production of inflammatory cytokines known as inflammaging characterized by systemic low grade persistent inflammation. In some cases, following viral infection, the inflammaging is characterized by an exaggerated reaction termed 'cytokine storm' mediated by IL6. The result is extensive tissue damage on a large number of cells and tissues translated by the severity of the clinical image of COVID-19 in some patients. As an immunological conclusion, an incompetent response of the immune system is a major factor of susceptibility to COVID-19 mediated mainly by the physiological and cellular alterations accompanying its aging and gender nature.

\section{THE RESULT OF THE CLINICAL MANIFESTATION OF COVID-19 INFECTION}

The presence of a pathogen in an organism with a given immune capital leads to the possible appearance of a panoply of symptoms. In COVID-19, the clinical picture varies from the asymptomatic or paucisymptomatic to the appearance of very diverse symptoms. In addition to the dominant respiratory manifestations like cough and shortness of breath, other clinical manifestations including multiple organe failure were also observed. These extrarespiratory symptoms can be fever, acute cardiac injury, kidney failure, gut and liver function abnormalities, myalgia, gastro-intestinal symptoms as vomiting, diarrhea, and abdominal pain. The observation of this multiple organ damage in COVID-19 suggests an invasion of SARS-CoV-2 to these different organs. This is mainly due to the ACE2 expression pattern in various organs, tissues and cell types wich might permit SARS-CoV-2 entry and pathogenesis. According to L. Fang, congestive heart failure, chronic kidney disease, diabetes, pulmonary and cardiovascular diseases are the main diseases predisposing to COVID-19 and subsequent mortality [27]. The question here is: Are comorbidities just a predisposing factor for COVID-19 or can they also contribute to the creation of the final clinical picture of the disease?

Several experimental models of human diseases have shown the essential role played by ACE2 in the development and progression of these comorbidities [29]. Furthermore, it has been shown that the increased expression of ACE2 protects against HTA [18], diabetic retinopathy [35], and myocardial infarction [36]. We stipulate here that individuals develop clinically these comorbidities do not have enough sufficiently protective ACE2 expression coupled with physiological intolerance to these abnormalities. This kind of patients usually uses antihypertensive and anti-diabetic drugs. Notably, the most frequent comorbidities of COVID-19 patients are often treated by ACEI and ARBs which, in their turn, upregulates ACE2 mRNA expression [37], [38]. The latter can make individuals susceptible to COVID-19 by increased ACE2 receptor expression. Disadvantageously, treatment and its 
impact on onset and progression of the pandemic were not assessed in either study. In this context, we propose to differentiate the COVID-19 patients according to their 'comorbidites' status and to provide a theoretical model making the link between the comorbidity, the ACE2 linked susceptibility and the clinical image of the pandemic (Fig 1). We thus propose in this model that the absence of comorbidity justifies a powerful immunological ground, a protective over-expression of ACE2 coupled with a susceptibility to the SARS-CoV-2 (more receptors). The clinical result is generally an asymptomatic carrier capable of transmitting the virus. In the same way, the presence of an untreated comorbidity highlights the absence of a sufficient protective ACE2 expression coupled with a relative resistance to the $S A R S-C o V-2$ infection (limited number of receptors). The end result is a COVID-19 patient with mild or severe symptoms based on the initial viral load. This parameter is related to the nature of exposure to the virus as well as the way in which prevention precautions are implemented and their effectiveness. Finally, a treated comorbidity is the equivalent of an immunologically weak organism, a drugmediated ACE2 overexpression coupled with an increased susceptibility to the SARS-CoV-2 which leads to a severe clinical picture of COVID-19.

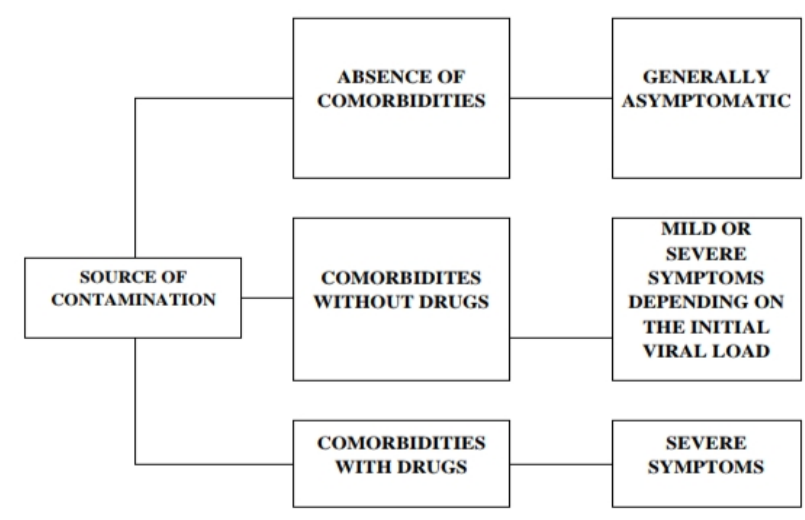

Fig. 1. Theoretical model of connection between the comorbidity previously presented and the clinical symptoms of COVID-19.

\section{CONCLUSION}

In summary, the worldwide struggle against the spread of COVID-19 and its repercussions on human health and economy does not seem to end soon. The spearhead in this fight is the social distancing given the unavailability of a safe and effective vaccine against the disease. Permanent monitoring of SARS-CoV-2 mutations, protection of the population at risk (in particular diabetics, hypertensives and those with an incompetent immune system), as well as the establishment of the links between the presence of comorbidities and the severity of the symptoms are elements which can reinforce the effectiveness of the control measures of COVID-19.

\section{REFERENCES}

[1] C. Huang, Y. Wang, X. Li, L. Ren, J, "Zhao, Y. Hu et al. Clinical features of patients infected with 2019 novel coronavirus in Wuhan, China, " Lancet, vol. 15;395(10223), pp. 497-506, 2020.

[2] P. Zhou, XL. Yang, KG. Wang, B. Hu, L, "Zhang, W. Zhang et al. A pneumonia outbreak associated with a new coronavirus of probable bat origin," Nature, vol. 579(7798), pp. 270-273, 2020.
[3] AE. Gorbalenya, SC. Baker, RS. Baric, RJ. De Groot, C. Drosten, AA. Gulyaeva et al, "The species Severe acute respiratory syndromerelated coronavirus: classifying $2019-\mathrm{nCoV}$ and naming it SARSCoV-2," Nat Microbiol, vol. 5, pp. 536-544, 2020.

[4] S. Su, G. Wong, W. Shi, J. Liu, ACK. Lai, J. Zhou et al, "Epidemiology, Genetic Recombination, and Pathogenesis of Coronaviruses," Trends Microbiol, vol. 24(6), pp. 490-502, 2016.

[5] A. Wu, Y. Peng, B. Huang, X. Ding, X. Wang, P. Niu et al, "Genome Composition and Divergence of the Novel Coronavirus (2019-nCoV) Originating in China," Cell Host Microbe. Vol. 27(3), pp. 325-328, 2020 .

[6] E. Mortola, P.Roy, "Efficient assembly and release of SARS coronavirus-like particles by a heterologous expression system," FEBS Lett, vol. 576(1-2), pp. 174-178, 2004.

[7] R. Yan, Y. Zhang, Y. Li, L. Xia, Y. Guo, Q. Zhou, "Structural basis for the recognition of SARS-CoV-2 by full-length human ACE2," Science, vol. 367(6485), pp. 1444-1448, 2020.

[8] R. Lu, X. Zhao, J. Li, P. Niu, B. Yang, H. Wu et al, "Genomic characterisation and epidemiology of 2019 novel coronavirus: implications for virus origins and receptor binding," Lancet, vol. 22, pp. 565-574, 2020.

[9] Y. Wan, J. Shang, R. Graham, RS. Baric, F. Li, "Receptor recognition by novel coronavirus from Wuhan: An analysis based on decade-long structural studies of SARS," J. Virol, vol. 94(7), e00127-20, 2020.

[10] L. Zhang, FM. Shen, F. Chen, Z. Lin, "Origin and evolution of the 2019 novel coronavirus," Clin Infect Dis, pii: ciaa112. doi: 10.1093/cid/ciaa112, 2020. [Epub ahead of print].

[11] M. Ciotti, S. Angeletti, M. Minieri, M. Giovannetti, D. Benvenuto, S. Pascarella et al, "COVID-19 Outbreak: An Overview," Chemotherapy, vol. 7, pp. 1-9, 2020.

[12] IN. Abdullahi, AU. Emeribe, JO. Mustapha, SA. Fasogbon, IB. Ofor, IS Opeyemi et al, "Exploring the genetics, ecology of SARS-COV-2 and climatic factors as possible control strategies against COVID-19," Infez Med, vol. 28(2), pp. 166-173, 2020.

[13] RA. Stein, "The 2019 coronavirus: Learning curves, lessons, and the weakest link," Int J Clin Pract, vol. 74(4):e13488, 2020.

[14] W. Ren, X. Qu, W. Li, Z. Han, M. Yu, P. Zhou et al, "Difference in receptor usage between severe acute respiratory syndrome (SARS) coronavirus and SARS-like coronavirus of bat origin," J. Virol, vol. 82, pp. 1899-1907, 2008.

[15] W. Li, C. Zhang, J. Sui, JH. Kuhn, MJ. Moore, S. Luo et al, "Receptor and viral determinants of SARS-coronavirus adaptation to human ACE2," EMBO J, vol. 24(8), pp. 1634-1643, 2005.

[16] D. Benvenuto, M. Giovanetti, M. Salemi, M. Prosperi, C. De Flora, LC. Junior Alcantara et al, "The global spread of 2019-nCoV: a molecular evolutionary analysis," Pathog Glob Health, vol. 114(2), pp. 64-67, 2020.

[17] M. Hussain, N. Jabeen, F. Raza, S. Shabbir, AA. Baig, A. Amanullah et al, "Structural variations in human ACE2 may influence its binding with SARS-CoV-2 spike protein," J Med Virol, 2020. doi: 10.1002/jmv.25832. [Epub ahead of print].

[18] M. Gheblawi, K. Wang, A. Viveiros, Q. Nguyen, JC. Zhong, AJ. Turner et al, "Angiotensin Converting Enzyme 2: SARS-CoV-2 Receptor and Regulator of the Renin-Angiotensin System," Circ Res, 2020. doi: 10.1161/CIRCRESAHA.120.317015. [Epub ahead of print].

[19] C. Vickers, P. Hales, V. Kaushik, L. Dick, J. Gavin, J. Tang et al, "Hydrolysis of biological peptides by human angiotensin-converting enzyme-related carboxypeptidase," J Biol Chem, vol. 277(17), pp. 14838-14843, 2002.

[20] W. Wang, S. Bodiga, SK. Das, J. Lo, V. Patel, GY. Oudit, "Role of ACE2 in diastolic and systolic heart failure," Heart Fail Rev, vol. 17(4-5), pp. 683-691, 2012.

[21] MC. Chappell, AC. Marshall, EM. Alzayadneh, HA. Shaltout, DI. Diz, "Update on the Angiotensin converting enzyme 2-Angiotensin (1-7)-MAS receptor axis: fetal programing, sex differences, and intracellular pathways," Front Endocrinol, vol. 4, pp. 201, 2014.

[22] X. Zou, K. Chen, J. Zou, P. Han, J. Hao, Z. Han, "Single-cell RNAseq data analysis on the receptor ACE2 expression reveals the potential risk of different human organs vulnerable to $2019-\mathrm{nCoV}$ infection," Front Med, 2020. doi: 10.1007/s11684-020-0754-0. [Epub ahead of print].

[23] H. Zhang, Z. Kang, G. Haiyi, D. Xu, J. Wang, Z. Li et al, "The digestive system is a potential route of 2019-nCov infection: a bioinformatics analysis based on single-cell transcriptomes," 2020. Preprint

https://www.biorxiv.org/content/10.1101/2020.01.30.927806v1.

[24] H. Xu, L. Zhong, J. Deng, J. Peng, H. Dan, X. Zeng et al, "High expression of ACE2 receptor of 2019-nCoV on the epithelial cells of 
oral mucosa," Int $J$ Oral Sci, vol. $12: 8,2020$. https://doi.org/10.1038/s41368-020-0074-x.

[25] M. Hoffmann, H. Kleine-Weber, S. Schroeder, N. Krüger, T. Herrler, S. Erichsen et al, "SARS-CoV-2 Cell Entry Depends on ACE2 and TMPRSS2 and Is Blocked by a Clinically Proven Protease Inhibitor," Cell, vol. 181(2), pp. 271-280, 2020.

[26] LM. Burrell, J. Risvanis, E. Kubota, RG. Dean, PS. MacDonald, S. $\mathrm{Lu}$ et al, "Myocardial infarction increases ACE2 expression in rat and humans," Eur Heart J, vol. 26(4), pp. 369-375, 2005.

[27] L. Fang, G. Karakiulakis, M. Roth, "Are patients with hypertension and diabetes mellitus at increased risk for COVID-19 infection?," Lancet Respir Med, vol. 8(4):e21, 2020.

[28] NE. Clarke, AJ. Turner, "Angiotensin-converting enzyme 2: the first decade," Int $J$ Hypertens, vol. 2012:307315. 2012. doi: $10.1155 / 2012 / 307315$.

[29] VB. Patel, JC. Zhong, MB. Grant, GY. Oudit, "Role of the ACE2/Angiotensin 1-7 Axis of the Renin-Angiotensin System in Heart Failure," Circ Res, vol. 118(8), pp. 1313-1326, 2016.

[30] VB. Patel, S. Bodiga, R. Basu, SK. Das, W. Wang, Z. Wang et al, "Loss of angiotensin-converting enzyme-2 exacerbates diabetic cardiovascular complications and leads to systolic and vascular dysfunction: a critical role of the angiotensin II/AT1 receptor axis," Circ Res, vol. 110(10), pp. 1322-1335, 2012.

[31] N. Chen, M. Zhou, X. Dong, J. Qu, F. Gong, Y. Han et al, "Epidemiological and clinical characteristics of 99 cases of 2019 novel coronavirus pneumonia in Wuhan, China: a descriptive study," Lancet, vol. 395(10223), pp. 507-513, 2020.

[32] A. Ruggieri, S. Anticoli, A. D'Ambrosio, L. Giordani, M. Viora, "The influence of sex and gender on immunity, infection and vaccination," Ann Ist Super Sanita. Vol. 52(2), pp. 198-204, 2016.

[33] B. Pinto, A. Oliveira, Y. Singh, L. Jimenez, A. Goncalves, R. Ogava et al, "ACE2 Expression is Increased in the Lungs of Patients with Comorbidities Associated with Severe COVID-19," 2020 https://www.medrxiv.org/content/10.1101/2020.03.21.20040261v1.

[34] K. Chen, J. Bi, Y. Su, MC. Chappell, JC, "Rose. Sex-Specific Changes in Renal Angiotensin-Converting Enzyme and AngiotensinConverting Enzyme 2 Gene Expression and Enzyme Activity at Birth and Over the First Year of Life," Reprod Sci, vol. 23(2), pp. 200-210, 2016.

[35] YP. Jarajapu, AD. Bhatwadekar, S. Caballero, S. Hazra, V. Shenoy, R. Medina et al, "Activation of the ACE2/angiotensin-(1-7)/Mas receptor axis enhances the reparative function of dysfunctional diabetic endothelial progenitors," Diabetes, vol. 62(4), pp. 1258-1269, 2013.

[36] Z. Kassiri, J. Zhong, D. Guo, R. Basu, X. Wang, PP. Liu et al, "Loss of angiotensin-converting enzyme 2 accelerates maladaptive left ventricular remodeling in response to myocardial infarction," Circ Heart Fail, vol. 2(5), pp. 446-455, 2009.

[37] Y. Ishiyama, PE. Gallagher, DB. Averill, EA. Tallant, KB. Brosnihan, CM. Ferrario, "Upregulation of angiotensin-converting enzyme 2 after myocardial infarction by blockade of angiotensin II receptors," Hypertension. Vol. 43(5), pp. 970-976, 2004.

[38] D. Gurwitz, "Angiotensin receptor blockers as tentative SARS-CoV-2 therapeutics," Drug Dev Res, 2020; doi: 10.1002/ddr.21656. [Epub ahead of print].

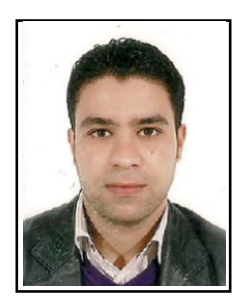

\section{Otmane EL BRINI}

Administrator, Ministry of Health, Morocco. $\mathrm{PhD}$, Ibn Tofail University, Morocco. 